\title{
On $x$-magnetic Surfaces Generated by Trajectory of $x$-magnetic Curves in Null Cone
}

\author{
Fatma ALMAZ *1 and Mihriban ALYAMAÇ KÜLAHCI ${ }^{2}$ \\ 1,2 Department of Mathematics, Firat University, 23119 ELAZIĞ/TÜRKIYE \\ ${ }^{1}$ fb_fat_almaz@hotmail.com, ${ }^{2}$ mihribankulahci@gmail.com
}

\begin{abstract}
In this work, we examine the impact of magnetic fields on the moving particle trajectories by variational approach to the magnetic flow associated with the Killing magnetic field on 2-dimensional lightlike cone $\mathbf{Q}^{2} \subset E_{1}^{3}$. We give some characterizations for $x$-magnetic curve and $x$-magnetic surface of rotation using the Killing magnetic field of this curve in $\mathbf{Q}^{2}$ and we give the different types of axes of rotation, then creates three different types of magnetic surfaces of rotation in 2-dimensional lightlike cone $\mathbf{Q}^{2} \subset E_{1}^{3}$.
\end{abstract}

Keywords: Killing vector field, lightlike cone, magnetic curve, magnetic surface, $W_{x}$ trajectory. 2010 MSC No: 53B30, 53B50, 53C80

\section{Introduction}

Although we know much about the submanifolds of the pseudo-Riemannian space forms, we have very few papers on submanifolds of the pseudo-Riemannian lightlike cone. A simply connected Riemannian manifold of dimension $n \geq 3$ is conformally flat if and only if it can be isometrically immersed as a hypersurface of the lightlike cone and the Killing vector fields define the Killing magnetic fields. Working on the degenerate submanifolds of Lorentzian manifolds with degenerate metric provides us with meaningful relavance between null submanifolds and spacetime, 1]. Barros and Romeo proved in [2, 3], that if $\left(M, g^{*}\right)$ has constant curvature, then the magnetic curves corresponding to a Killing magnetic field are centerlines of Kirchhoffelastic rods. V.N. Mishra and at al studied different metrics in different ambiant spaces [6, 15, 16, 18, 19, 20]. In [21, the author defined magnetic curves on a Riemannian manifold $\left(M, g^{*}\right)$ according trajectories of charged particles moving on $M$ under the action of a magnetic field $F_{*}$. In [4], Bozkurt et al. invastigated the magnetic flow associated with the Killing magnetic field in a three-dimensional oriented Riemann manifold $\left(M^{3}, g^{*}\right)$. In [12, the author studied cone curves and investigate the notations of the cone curvature function and also gave some examples of cone curves in Minkowski space. In [5], they characterized the Killing vector fields on a Walker manifold $M_{f}^{3}$ aiming to obtain the corresponding Killing magnetic curves and characterized the normal magnetic curves corresponding to some Killing vector fields on $M_{f}^{3}$, obtaining their explicit expressions for certain functions $f$. In [7, the authors investigated magnetic curves corresponding to the Killing magnetic field $W$ in the 3-dimensional Minkowski space. In [8], they determined all magnetic curves corresponding to the Killing magnetic fields on the 3-dimensional Euclidean space. In [13], the author gave the representation formulas of the curves in $\mathbf{Q}^{2}$ and $\mathbf{Q}^{3}$ and defined the functions of the cone curves

\footnotetext{
${ }^{*}$ Corresponding author. Fatma ALMAZ ${ }^{1}$ fb_fat_almaz@hotmail.com
} 


\section{Preliminaries}

Let $E_{1}^{3}$ be the 3 -dimensional pseudo-Euclidean space with the

$$
g^{*}(V, W)=\langle V, W\rangle=v_{1} w_{1}+v_{2} w_{2}-v_{3} w_{3}
$$

for all $V=\left(v_{1}, v_{2}, v_{3}\right), W=\left(w_{1}, w_{2}, w_{3}\right) \in E_{1}^{3} . E_{1}^{3}$ is a flat pseudo-Riemannian manifold of signature $(2,1)$.

Let $M$ be a submanifold of $E_{1}^{3}$. If the pseudo-Riemannian metric $g^{*}$ of $E_{1}^{3}$ induces a pseudo-Riemannian metric $\widetilde{g}$ (respectively, a Riemannian metric, a degenerate quadratic form) on $M$, then $M$ is called a timelike ( respectively, spacelike, degenerate) submanifold of $E_{1}^{3}$. The lightlike cone is defined by

$$
\mathbf{Q}^{2}=\left\{x \in E_{1}^{3}: g^{*}(x, x)=0\right\}
$$

Let $E_{1}^{3}$ be 3 -dimensional Minkowski space and $\mathbf{Q}^{2}$ be the lightlike cone in $E_{1}^{3}$. A vector $W \neq 0$ in $E_{1}^{3}$ is called spacelike, timelike or lightlike, if $\langle W, W\rangle>0,\langle W, W\rangle<0$ or $\langle W, W\rangle=0$, respectively. A frame field $\{x, \alpha, y\}$ on $E_{1}^{3}$ is called an asymptotic orthonormal frame field, if

$$
\langle x, x\rangle=\langle y, y\rangle=\langle x, \alpha\rangle=\langle y, \alpha\rangle=0,\langle x, y\rangle=\langle\alpha, \alpha\rangle=1 .
$$

We assume that curve $x: I \rightarrow \mathbf{Q}^{2} \subset E_{1}^{3}$ is a regular curve in $\mathbf{Q}^{2}$ for $t \in I$. In the following, we always assume that the curve is regular.

Using $x^{\prime}(s)=\alpha(s)$, from an asymptotic orthonormal frame along the curve $x(s)$ and the cone Frenet formulas of $x(s)$ are given by

$$
\begin{aligned}
x^{\prime}(s) & =\alpha(s) \\
\alpha^{\prime}(s) & =\kappa(s) x(s)-y(s) \\
y^{\prime}(s) & =-\kappa(s) \alpha(s)
\end{aligned}
$$

where the function $\kappa(s)$ is called cone curvature function of the curve $x(s)$, [12]. The Lorentzian cross-product $\times: E_{1}^{3} \times E_{1}^{3} \rightarrow E_{1}^{3}$ is defined by the formula

$$
V \times W=\left[\begin{array}{ccc}
i & j & -k \\
v_{1} & v_{2} & v_{3} \\
w_{1} & w_{2} & w_{3}
\end{array}\right]
$$

where $V=\left(v_{1}, v_{2}, v_{3}\right), W=\left(w_{1}, w_{2}, w_{3}\right) \in E_{1}^{3}$. Here $i, j, k$ have usual meaning. We say that this product has similar algebraic properties as the cross product in $E^{3}$. Hence, it is skew-symmetric and $V \times W$ is ortogonal on both $V$ and $W$. The Lorentz force $\psi$ of a magnetic field $F_{*}$ on $\mathbf{Q}^{2}$ is defined to be a skew-symetric operator given by

$$
g^{*}(\psi(V), W)=F_{*}(V, W)
$$

for all $V, W \in \mathbf{Q}^{2}$. The $\alpha$-magnetic trajectories of $F_{*}$ are $x$ on $\mathbf{Q}^{2}$ that satisfy the Lorentzian equation

$$
\nabla_{x^{\prime}} x^{\prime}=\psi\left(x^{\prime}\right)
$$

Furthermore, the mixed product of the vector fields $V, W, Z \in \mathbf{Q}^{2}$ is the defined by

$$
g^{*}(V \times W, Z)=d v_{g^{*}}(V, W, Z),
$$

where $d v_{g^{*}}$ denotes a volume on $\mathbf{Q}^{2}$. If $W$ is a Killing vector in $\mathbf{Q}^{2}$ and let $F_{* W}=\imath_{W} v o l_{g^{*}}$ be the corresponding Killing magnetic field, here the inner product is indicated by $\imath$. Hence the equation Lorentz force of $F_{* W}$ is

$$
\psi(X)=W \times X
$$

for all $X \in \mathbf{Q}^{2}$. Corresponding the Lorentz equation can be written as

$$
\nabla_{x^{\prime}} x^{\prime}=\psi\left(x^{\prime}\right)=W \times x^{\prime} .
$$

In Minkowski space $E_{1}^{3}$, consider the Killing vector field $W=a \partial_{x}+b \partial_{y}+c \partial_{z}$, with $a, b, c \in \mathbb{R}$, the magnetic trajectories $x: I \rightarrow \mathbf{Q}^{2} \subset E_{1}^{3}$ determined by $W$ are solutions of the Lorentz equation

$$
x^{\prime \prime}=W \times x^{\prime},
$$


Definition 2.1. A one-parameter group of diffeomorphisms of a manifold $M$ is a smooth map $\psi: M \times \mathbb{R} \rightarrow M$, such that $\psi_{t}(x)=\psi(x, t)$, where

$\psi_{t}: M \rightarrow M$ is a diffeomorephism,

$\psi_{0}=i d$.

$\psi_{s+t}=\psi_{s} o \psi_{t}$.

This group is accociated with a vector field $\mathrm{V}$ given by $\frac{d}{d t} \psi_{t}(x)=W(x)$, and the group of diffeomorphisms is called the flow of $W$. If a one-parameter group of isometries is generated by a vector field $W$, then this vector field is called a Killing vector field, [14.

\section{$3 x$-magnetic Curves in the Null Cone $\mathbf{Q}^{2} \subset \mathbf{E}_{1}^{3}$}

In this section, we give some characterizations for $x$-magnetic curve and surface of rotation using the Killing magnetic field of this curve in $\mathbf{Q}^{2} \subset E_{1}^{3}$.

Definition 3.1. Let $x: I \rightarrow \mathbf{Q}^{2} \subset E_{1}^{3}$ be a spacelike curve in $\mathbf{Q}^{2}$ and $F_{* W}$ be a magnetic field on $\mathbf{Q}^{2} \subset E_{1}^{3}$. We call the curve $x$ a $x$-magnetic curve if its $W_{x}$ vector field satisfies the Lorentz force equation

$$
\nabla_{x^{\prime}} x=\nabla_{\alpha} x=\psi^{x}(x)=W_{x} \times x .
$$

Theorem 3.2. Let $x(s)$ be a unit speed spacelike $x$-magnetic curve in the $\mathbf{Q}^{2} \subset E_{1}^{3}$ with the asymptotic orthonormal frame $\{x, \alpha, y\}$. The Lorentz force in the Frenet frame are given as follows

$$
\psi^{x}=\left[\begin{array}{ccc}
0 & 1 & 0 \\
w_{3} & 0 & -1 \\
0 & -w_{3} & 0
\end{array}\right]
$$

where $w_{3}$ is a function defined by $w_{3}=g^{*}\left(\psi^{x}(\alpha), y\right)$.

Proof 3.3. Let $x(s)$ be a unit speed spacelike $x$-magnetic curve in the $\mathbf{Q}^{2} \subset E_{1}^{3}$ with the asymptotic orthonormal frame $\{x, \alpha, y\}$. From the definition of the magnetic curve and equation (2.1), we know that

$$
\psi^{x}(x)=\vec{\alpha}
$$

Furthermore, since $\psi^{x}(\alpha) \in \operatorname{Span}\{x, \alpha, y\}$, we can write

$$
\psi^{x}(\alpha)=A_{1} \vec{x}+B_{1} \vec{\alpha}+C_{1} \vec{y} .
$$

By using the following equalities

$$
\begin{aligned}
& A_{1}=g^{*}\left(\psi^{x}(\alpha), y\right)=w_{3} ; B_{1}=g^{*}\left(\psi^{x}(\alpha), \alpha\right)=0 \\
& C_{1}=g^{*}\left(\psi^{x}(\alpha), x\right)=-g^{*}\left(\psi^{x}(x), \alpha\right)=-g^{*}(\alpha, \alpha)=-1
\end{aligned}
$$

we get

$$
\psi^{x}(\alpha)=w_{3} \vec{x}-\vec{y}
$$

Similarly, we can easily obtain

$$
\psi^{x}(y)=-w_{3} \vec{\alpha}
$$

Theorem 3.4. Let $x(s)$ be a unit speed spacelike $x$-magnetic curve in the $\mathbf{Q}^{2} \subset E_{1}^{3}$. The curve $x$ is then a $x$-magnetic trajectory of a magnetic vector field $W_{x}$ if and only if the vector field $W_{x}$ can be written along the curve $x$ as the following

$$
W_{x}(s)=\overrightarrow{x(s)}+\overrightarrow{y(s)}
$$


Proof 3.5. Let $x(s)$ be a unit speed spacelike $x$-magnetic curve with $x$-magnetic trajectory of a magnetic field $W_{x}$. Using theorem 1 and definition 1, we can easily obtain that

$$
W_{x}(s)=\overrightarrow{x(s)}+\overrightarrow{y(s)}
$$

Conversely, we assume that equation (3.2) holds. Then we get $\psi^{x}(x)=W_{x} \times x$. Therefore the curve $x$ is a $x-$ magnetic trajectory of the magnetic vector field $W_{x}$.

Theorem 3.6. Let $x$ be a $x$-magnetic trajectory according to the Killing vector field $W_{x}=\vec{x}+\vec{y}$ in $\mathbf{Q}^{2} \subset E_{1}^{3}$. Then the curve $x$ can be expressed as follows

$$
x_{x}(s)=x(0)+c W_{x}
$$

where $c \in \mathbb{R}_{0}$.

Proof 3.7. We prove the theorem according to the $W_{x}$. Since $W_{x}$ and $x(0)$ are linearly independent and $W_{x}$ is spacelike. We consider $W_{x}, W_{x} \times x(0)$ and $W^{*}$ be linearly independent and satisfy

$$
\left\langle W_{x}, W^{*}\right\rangle=0,\left\langle W_{x}, W_{x} \times x(0)\right\rangle=0,\left\langle W^{*}, W_{x} \times x(0)\right\rangle=0 .
$$

So, we can take

$$
W^{*}=2 x(0)-\left\langle W_{x}, x(0)\right\rangle W_{x} .
$$

We can write

$$
\begin{aligned}
x(s) & =x(0)+\lambda_{x}(s) W_{x}+\mu_{x}(s) W_{x} \times x(0)+\rho_{x}(s) W^{*}, \\
x^{\prime}(s) & =x^{\prime}(0)+\lambda_{x}^{\prime}(s) W_{x}+\mu_{x}^{\prime}(s) W_{x} \times x(0)+\rho_{x}^{\prime}(s) W^{*},
\end{aligned}
$$

where $\lambda_{x}(s), \mu_{x}(s), \rho_{x}(s), \lambda_{x}^{\prime}(s), \mu_{x}^{\prime}(s), \rho_{x}^{\prime}(s)$ functions satisfied the following

$$
\lambda_{x}(0)=0, \mu_{x}(0)=0, \rho_{x}(0)=0, \lambda_{x}^{\prime}(0)=0, \mu_{x}^{\prime}(0)=0, \rho_{x}^{\prime}(0)=0,
$$

where $s=0$. The Lorentz equation $x^{\prime}(s)=W_{x} \times x(s)$ can be written as

$$
x^{\prime}(0)+\lambda_{x}^{\prime}(s) W_{x}+\mu_{x}^{\prime}(s) W_{x} \times x(0)+\rho_{x}^{\prime}(s) W^{*}=W_{x} \times x(0)-\mu_{x}(s) W^{*}-\rho_{x}(s) W_{x} \times x(0),
$$

since $x^{\prime}(0)=W_{x} \times x(0)$ for $s=0$, we have

$$
0=\lambda_{x}^{\prime}(s) W_{x}+\left(\mu_{x}^{\prime}(s)+\rho_{x}(s)\right) W_{x} \times x_{x}(0)+\left(\mu_{x}(s)+\rho_{x}^{\prime}(s)\right) W^{*},
$$

which is equivalent to

$$
\lambda_{x}^{\prime}(s)=0, \mu_{x}^{\prime}(s)+\rho_{x}(s)=0, \mu_{x}(s)+\rho_{x}^{\prime}(s)=0 .
$$

Solving the previous differential equations and using the initial conditions (3.4), we get

$$
\lambda_{x}(s)=c, \mu_{x}(s)=0, \rho_{x}(s)=0,
$$

where $c \in \mathbb{R}_{0}$. Hence the curve $x$ is written as $x_{x}(s)=x(0)+c W_{x}$.

\section{Surface of Rotation Generated by The Lorentz force $\psi^{x}$ with $x$-magnetic curve}

Theorem 4.1. Let $x$ be a $x$-magnetic trajectory according to the killing vector field $W_{x}=\mp \kappa(\vec{x}+\vec{y})$ in $\mathbf{Q}^{2} \subset E_{1}^{3}$. Then the following statements holds $\left.i\right) \Delta_{1}^{x}(s, t)$ surface of rotation generated by the $\psi^{x}$ can be expressed as follow

$$
\Delta_{1}^{x}(s, t)=\left(\begin{array}{c}
\left(\frac{1+\cosh \left(\sqrt{2 w_{3}} s\right)}{2}+\frac{1-\cosh \left(\sqrt{2 w_{3}} s\right)}{2 w_{3}}\right)(t+b), \\
\frac{w_{3}-1}{\sqrt{w_{3}}} \sin \left(\sqrt{w_{3}} s\right), \\
\left(\frac{w_{3}\left(1-\cosh \left(\sqrt{2 w_{3}} s\right)\right)}{2}+\frac{1+\cosh \left(\sqrt{2 w_{3}} s\right)}{2}\right)(t+b)
\end{array}\right)
$$


and the surface of revolution $\Delta_{1}^{x}$ with $x_{1}(t)=(t+b, 0, t+b), b \in \mathbb{R}_{0}$ and $-\infty<s<\infty, t \in I \subset \mathbb{R}$.

$i_{1}$ ) If $w_{3}=$ constant, the surface of revolution $\Delta_{1}^{x}$ with $x_{1}(t)$ is minimal surface and the Gaussian and mean curvatures are $K, H=0$.

$i_{2}$ ) If $w_{3} \neq$ constan $t$ for $-\infty<s<\infty, t \in I \subset \mathbb{R}$ and for $s, t=0$, the Gaussian and mean curvatures of the surface of revolution $\Delta_{1}^{x}$ with $x_{1}(t)$ are

$$
\begin{gathered}
K=b \sqrt{2}\left(\begin{array}{c}
w_{3}^{\prime \prime}\left(w_{3}-1\right)-2 w_{3}^{\prime}\left(\frac{w_{3}-1}{\sqrt{w_{3}}}\right)^{\prime} \sqrt{w_{3}} \\
\left(2\left(w_{3}-1\right)^{2}-w_{3}^{\prime 2} b^{2}\right)\left(1-w_{3}^{2}\right)-\left(w_{3} w_{3}^{\prime} b\right)^{2}
\end{array}\right) \\
H=\frac{-\sqrt{2}}{2} \frac{\left(\begin{array}{c}
w_{3}^{\prime}\left(w_{3}-1\right)\left(1-w_{3}^{2}\right) \\
+b\left(\begin{array}{c}
\left(2\left(w_{3}-1\right)^{2}-w_{3}^{\prime 2} b^{2}\right) \\
\left(-2\left(\frac{w_{3}-1}{\sqrt{w_{3}}}\right)^{\prime} w_{3}^{\prime} \sqrt{w_{3}}+w_{3}^{\prime \prime}\left(w_{3}-1\right)\right)
\end{array}\right)
\end{array}\right)}{\left(2\left(w_{3}-1\right)^{2}-w_{3}^{\prime 2} b^{2}\right)\left(1-w_{3}^{2}\right)-\left(w_{3} w_{3}^{\prime} b\right)^{2}}
\end{gathered}
$$

$\left.i_{3}\right)$ The condition of minimal surface of revolution $\Delta_{2}^{x}$ with $x_{2}(t)$ is

$$
\begin{aligned}
& w_{3}^{\prime \prime} b\left(w_{3}-1\right)\left(w_{3}^{\prime 2} b^{2}-2\left(w_{3}-1\right)^{2} w_{3}\right)+b w_{3}^{\prime 2}\left(2\left(w_{3}-1\right)^{2}-w_{3}^{\prime 2} b^{2}\right) \\
& =w_{3}^{\prime} w_{3}\left(w_{3}-1\right)\left(1-w_{3}^{2}\right)
\end{aligned}
$$

for $s, t=0$.

ii) $\Delta_{2}^{x}(s, t)$ surface of rotation generated by the $\psi^{x}$ can be expressed as follow

$$
\Delta_{2}^{x}(s, t)=\left(\begin{array}{c}
\frac{\sinh \left(\sqrt{2 w_{3}} s\right)(t+b)}{2 \sqrt{w_{3}}}, \\
\frac{\left(3-\cosh \left(\sqrt{2 w_{3}} s\right)\right)(t+b)}{2}, \\
\frac{-\sqrt{2 w_{3}} \sinh \left(\sqrt{2 w_{3}} s\right)(t+b)}{2}
\end{array}\right)
$$

where $\kappa$ is the curvature of the curve $x$ and $x_{2}(t)=(t+b, 0, t+b), b \in \mathbb{R}_{0}$ and $-\infty<s<\infty, t \in I \subset \mathbb{R}$.

$\left.i i_{1}\right)$ the Gaussian and mean curvatures are given by

$$
\begin{aligned}
K & =-\frac{(t+b)^{2} w_{3}^{2} \cosh ^{4}\left(\sqrt{2 w_{3}} s\right)}{2 \xi} \\
H & =-\frac{(t+b)^{2} \sqrt{w_{3}} \cosh ^{2}\left(\sqrt{2 w_{3}} s\right)\left(\sinh \left(2 \sqrt{2 w_{3}} s\right)\right.}{\left.4 w_{3}-2 w_{3}^{2}+1-6 w_{3} \sinh \left(\sqrt{2 w_{3}} s\right)\right)}
\end{aligned}
$$

where $w_{3}=$ cons $\tan t$ and

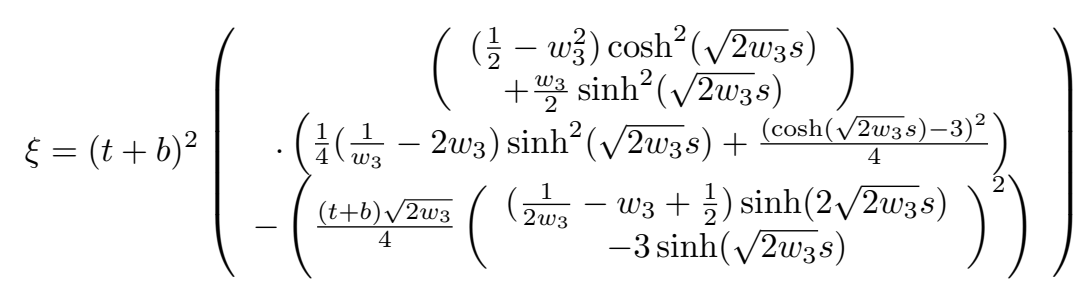

$\left.i_{2}\right)$ The condition of minimal surface of revolution $\Delta_{2}^{x}$ with $x_{2}(t)$ is

$$
\cosh \left(\sqrt{2 w_{3}} s\right)=\frac{3 w_{3}}{1+w_{3}-2 w_{3}^{2}} .
$$

Proof 4.2. we look for a one parameter group of Lorentz of transformation which fixed all points on the xy-plane. This requires the Killing vector field to satisfy $W_{x}(s)=\overrightarrow{x(s)}+\overrightarrow{y(s)}$. Hence, we can use a $3 \times 3$ matrix $\psi^{x}$. Now, we have the one parameter group of homomorphism $\psi_{s}(x, \alpha, y)$ given by $\psi_{s}^{\prime}(x)=\psi^{x} \psi_{s}(x)$. So, we obtain $\psi_{s}(x)=e^{s \psi_{s}} x$ and calculating the matrix exponential, we have

$$
\Pi^{x}(s)=\left[\begin{array}{ccc}
\frac{1+\cosh \left(\sqrt{2 w_{3}} s\right)}{2} & \frac{\sinh \left(\sqrt{2 w_{3}} s\right)}{\sqrt{2 w_{3}}} & \frac{1-\cosh \left(\sqrt{2 w_{3}}\right) s}{2 w_{3}} \\
\sqrt{w_{3}} \sin \left(\sqrt{w_{3}} s\right) & \frac{3-\cosh \left(\sqrt{2 w_{3}} s\right)}{2} & \frac{-\sin \left(\sqrt{w_{3}} s\right)}{\sqrt{w_{3}}} \\
\frac{w_{3}\left(1-\cosh \left(\sqrt{2 w_{3}} s\right)\right)}{2} & \frac{-\sqrt{2 w_{3}} \sinh \left(\sqrt{2 w_{3}} s\right)}{2} & \frac{1+\cosh \left(\sqrt{2 w_{3}} s\right)}{2}
\end{array}\right]
$$


where $-\infty<s<\infty$. By the Lorentz force $\psi^{x}$ rotation for $x$-magnetic curve we mean; rotating a curve using the rotation matrix (3.1), the plane of rotation is given by $W_{x}(s)=\overrightarrow{x(s)}+\overrightarrow{y(s)}$. Clearly, any point in $\mathbf{Q}^{2}$ can be carried to the $x y$-plane by some notation, so we assume that the $x$ curve lies in $x y$-plane. Hence, one of its parametrizations is

$$
x_{1}(t)=(t+b, 0, t+b), b \in \mathbb{R}_{0} .
$$

Hence, the surface of revolution $W_{x} \Delta_{1}^{x}$ around $W_{x}$ can be parametrized as follow:

$$
\Delta_{1}^{x}(s, t)=\left(\begin{array}{c}
\left(\frac{1+\cosh \left(\sqrt{2 w_{3}} s\right)}{2}+\frac{1-\cosh \left(\sqrt{2 w_{3}} s\right)}{2 w_{3}}\right)(t+b), \\
\frac{w_{3}-1}{\sqrt{w_{3}}} \sin \left(\sqrt{w_{3}} s\right), \\
0 \\
\left(\frac{w_{3}\left(1-\cosh \left(\sqrt{2 w_{3}} s\right)\right)}{2}+\frac{1+\cosh \left(\sqrt{2 w_{3}} s\right)}{2}\right)(t+b)
\end{array}\right),
$$

where $-\infty<s<\infty, t \in I$. We examined the surface of revolution $\Delta_{1}^{x}$ for $s \neq 0$. For the gaussian and mean curvatures, we have

$$
\begin{aligned}
E & =(t+b)^{2}\left(1-w_{3}\right)^{2} \sinh ^{2}\left(\sqrt{2 w_{3}} s\right)\left(\frac{1}{4 w_{3}}-\frac{w_{3}}{2}\right) \\
& \left.+\left(1-w_{3}\right)^{2} \cos ^{2}\left(\sqrt{w_{3}} s\right)\right) \\
F & =\left(\vartheta \frac{\left(w_{3}-1\right) \sqrt{2 w_{3}}}{2}\left(\frac{1}{w_{3}^{2}}-1\right) \sinh \left(\sqrt{2 w_{3}} s\right)(t+b)\right) \\
G & =\vartheta^{2}\left(\frac{1}{w_{3}^{2}}-1\right) ; N=0, M=0, L=0 ; \\
n_{\Lambda_{1}^{x}}= & \left(-\left(w_{3}-1\right) \cos \left(\sqrt{w_{3}} s\right) \vartheta, 0,-\left(w_{3}-1\right) \cos \left(\sqrt{w_{3}} s\right) \frac{\vartheta}{w_{3}}\right)
\end{aligned}
$$

where $\frac{\left(w_{3}+1\right)}{2}+\frac{\left(w_{3}-1\right)}{2} \cosh \left(\sqrt{2 w_{3}} s\right)=\vartheta$.

Thus, this results in the first fundemental form is $I_{\Delta_{1}^{x}}=E G-F^{2} \neq 0$, for $s \neq 0, K, H=0$. Hence, we say that the surface of revolution $\Delta_{1}^{x}$ with $x_{1}(t)$ is minimal surface.

For $s, t=0$ and the function $w_{3}(s)$, we have

$$
\begin{gathered}
E=2\left(1-w_{3}\right)^{2}-b^{2} w_{3}^{\prime 2}, \\
F=-b w_{3} w_{3}^{\prime} ; G=\left(1-w_{3}^{2}\right), \\
N=b \sqrt{2}\left(\begin{array}{c}
-2 w_{3}^{\prime} \sqrt{w_{3}}\left(\frac{w_{3}-1}{\sqrt{w_{3}}}\right)^{\prime} \\
+w_{3}^{\prime \prime}\left(w_{3}-1\right)
\end{array}\right), \\
M=0 ; L=\sqrt{2}\left(w_{3}-1\right) w_{3}^{\prime} ; \\
n_{\Lambda_{1}^{x}}=\left(-\sqrt{2} w_{3}\left(w_{3}-1\right),-w_{3}^{\prime},-\sqrt{2}\left(w_{3}-1\right)\right),
\end{gathered}
$$

using the previous equations, we can write

$\left.i_{1}\right)$ If $w_{3}=$ cons tan $t$, since the Gaussian and mean curvatures are $K, H=0$, the surface of revolution $\Delta_{1}^{x}$ with $x_{1}(t)$ is minimal surface.

$\left.i_{2}\right)$ If $w_{3} \neq$ cons $\tan t$, for $s, t=0$ the Gaussian and mean curvatures of the surface of revolution $\Delta_{1}^{x}$ with $x_{1}(t)$ are

$$
\begin{gathered}
K=b \sqrt{2}\left(\frac{w_{3}^{\prime \prime}\left(w_{3}-1\right)-2 w_{3}^{\prime}\left(\frac{w_{3}-1}{\sqrt{w_{3}}}\right)^{\prime} \sqrt{w_{3}}}{\left(2\left(w_{3}-1\right)^{2}-w_{3}^{\prime 2} b^{2}\right)\left(1-w_{3}^{2}\right)-\left(w_{3} w_{3}^{\prime} b\right)^{2}}\right) \\
H=\frac{-\sqrt{2}}{2} \frac{\left(\begin{array}{c}
w_{3}^{\prime}\left(w_{3}-1\right)\left(1-w_{3}^{2}\right) \\
+b\left(\begin{array}{c}
\left(2\left(w_{3}-1\right)^{2}-w_{3}^{\prime 2} b^{2}\right) \\
\left(-2\left(\frac{w_{3}-1}{\sqrt{w_{3}}}\right)^{\prime} w_{3}^{\prime} \sqrt{w_{3}}+w_{3}^{\prime \prime}\left(w_{3}-1\right)\right)
\end{array}\right)
\end{array}\right)}{\left(2\left(w_{3}-1\right)^{2}-w_{3}^{\prime 2} b^{2}\right)\left(1-w_{3}^{2}\right)-\left(w_{3} w_{3}^{\prime} b\right)^{2}} .
\end{gathered}
$$


The condition of minimal surface of revolution $\Delta_{1}^{x}$ with $x_{1}(t)$ is

$$
\begin{aligned}
& w_{3}^{\prime \prime} b\left(w_{3}-1\right)\left(w_{3}^{\prime 2} b^{2}-2\left(w_{3}-1\right)^{2} w_{3}\right)+b w_{3}^{\prime 2}\left(2\left(w_{3}-1\right)^{2}-w_{3}^{\prime 2} b^{2}\right) \\
& =w_{3}^{\prime} w_{3}\left(w_{3}-1\right)\left(1-w_{3}^{2}\right) .
\end{aligned}
$$

Let be $x$ curve as $x_{2}(t)=(0, t+b, 0), b \in \mathbb{R}_{0}$. Hence, using the axis of rotation $x_{2}(t)$, the surface of revolution $W_{x} \Delta_{2}^{x}$ around $W_{x}$ can be parametrized as follow:

$$
\begin{gathered}
\Delta_{2}^{x}(s, t)=\Pi^{x}(s) \times\left[\begin{array}{c}
0 \\
t+b \\
0
\end{array}\right] \\
\Delta_{2}^{x}(s, t)=\left(\begin{array}{c}
\frac{\sinh \left(\sqrt{2 w_{3}} s\right)(t+b)}{2 \sqrt{w_{3}}}, \\
\frac{3-\cosh \left(\sqrt{2 w_{3}} s\right)(t+b)}{2}, \\
\frac{-(t+b) \sqrt{2 w_{3}} \sinh \left(\sqrt{2 w_{3}} s\right)}{2}
\end{array}\right),
\end{gathered}
$$

For the gaussian and mean curvatures, we have

$$
\begin{gathered}
E=(t+b)^{2}\left(\begin{array}{c}
\left(\frac{1}{2}-w_{3}^{2}\right) \cosh ^{2}\left(\sqrt{2 w_{3}} s\right) \\
+\frac{w_{3}}{2} \sinh ^{2}\left(\sqrt{2 w_{3}} s\right)
\end{array}\right) \\
F=\frac{(t+b) \sqrt{2 w_{3}}}{4}\left(\begin{array}{c}
\left(\frac{1}{2 w_{3}}-w_{3}+\frac{1}{2}\right) \sinh \left(2 \sqrt{2 w_{3}} s\right) \\
-3 \sinh \left(\sqrt{2 w_{3}} s\right)
\end{array}\right) \\
G=\frac{1}{4}\left(\frac{1}{w_{3}}-2 w_{3}\right) \sinh ^{2}\left(\sqrt{2 w_{3}} s\right)+\frac{\left(\cosh \left(\sqrt{2 w_{3}} s\right)-3\right)^{2}}{4} \\
M=-\frac{\sqrt{2} w_{3}(t+b)}{2} \cosh ^{2}\left(\sqrt{2 w_{3}} s\right), N=0, L=0 \\
n_{\Delta_{2}^{x}}=\left(\begin{array}{c}
\frac{w_{3}(t+b)}{2}\left(1-3 \cosh \left(\sqrt{2 w_{3}} s\right)\right) \\
0 \\
\frac{-\sqrt{2}(t+b)}{4}\left(1-3 \cosh \left(\sqrt{2 w_{3}} s\right)\right)
\end{array}\right)
\end{gathered}
$$

this results in the first fundamental form and the second fundamental form are given as

$$
I_{\Delta_{2}^{x}}=\xi ; I I_{\Delta_{2}^{x}}=\frac{-w_{3}^{2}(t+b)^{2}}{2} \cosh ^{4}\left(\sqrt{2 w_{3}} s\right) .
$$

Therefore, the Gaussian and mean curvatures are given by

$$
\begin{aligned}
K & =\frac{-(t+b)^{2} w_{3}^{2} \cosh ^{4}\left(\sqrt{2 w_{3}} s\right)}{2 \xi} \\
H & =\frac{-\left(\begin{array}{c}
(t+b)^{2} \sqrt{w_{3}} \cosh ^{2}\left(\sqrt{2 w_{3}} s\right)\left(\sinh \left(2 \sqrt{2 w_{3}} s\right)\right. \\
\left(w_{3}-2 w_{3}^{2}+1-6 w_{3} \sinh \left(\sqrt{2 w_{3}} s\right)\right)
\end{array}\right)}{4 w_{3} \xi},
\end{aligned}
$$

where

$$
\begin{gathered}
\xi=(t+b)^{2}\left(\begin{array}{c}
\left(\frac{1}{2}-w_{3}^{2}\right) \cosh ^{2}\left(\sqrt{2 w_{3}} s\right) \\
+\frac{w_{3}}{2} \sinh ^{2}\left(\sqrt{2 w_{3}} s\right)
\end{array}\right) \\
\cdot\left(\frac{1}{4}\left(\frac{1}{w_{3}}-2 w_{3}\right) \sinh ^{2}\left(\sqrt{2 w_{3}} s\right)+\frac{\left(\cosh \left(\sqrt{2 w_{3}} s\right)-3\right)^{2}}{4}\right)- \\
\left(\frac{(t+b) \sqrt{2 w_{3}}}{4}\left(\begin{array}{c}
\left(\frac{1}{2 w_{3}}-w_{3}+\frac{1}{2}\right) \sinh \left(2 \sqrt{2 w_{3}} s\right) \\
-3 \sinh \left(\sqrt{2 w_{3}} s\right)
\end{array}\right)\right)^{2} .
\end{gathered}
$$

Hence, we say that the surface of revolution $\Delta_{2}^{x}$ with $x_{2}(t)$ is minimal surface for $\cosh \left(\sqrt{2 w_{3}} s\right)=\frac{3 w_{3}}{1+w_{3}-2 w_{3}^{2}}$. 


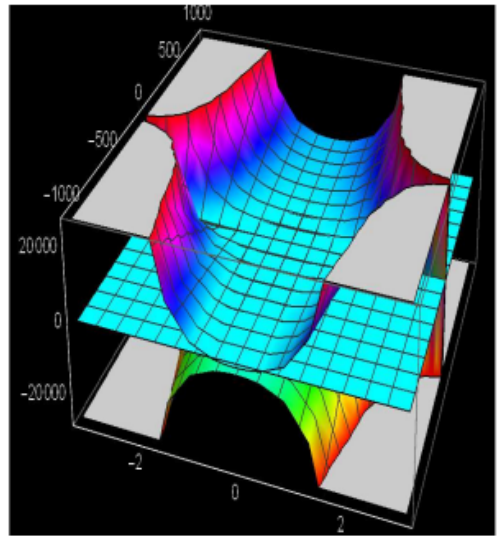

(a) The surface of $\Delta_{1}^{x}$

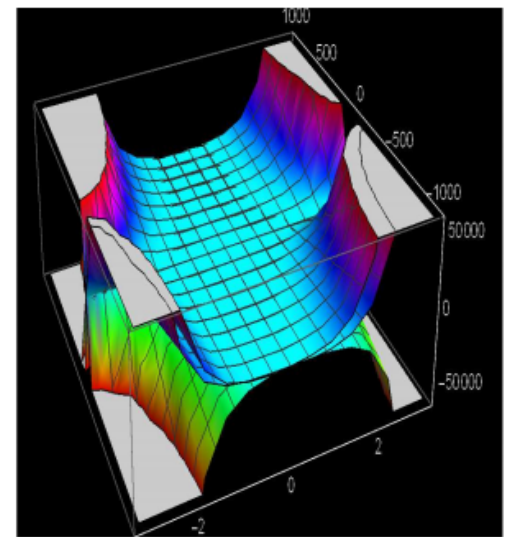

(b) The surface of $\Delta_{2}^{x}$

Figure 1: Graphics of surfaces $\Delta_{1}^{x}$ and $\Delta_{2}^{x}$

\section{References}

[1] A. Asperti, M. Dajezer, Conformally Flat Riemannian Manifolds as Hypersurface of the Light Cone, Canad. Math. Bull., 32, (1989), 281-285. https://doi.org/10.4153/cmb-1989-041-8

[2] M. Barros, M.F. Cabrerizo, A. Romero, Magnetic Vortex Filament Flows, J. Math. Phys., 48, (2007), 1-27. https://doi.org/10.1063/1.2767535

[3] M. Barros, A. Romero, Magnetic Vortices, Euro phys. Lett., 77, (2007), 1-5.

[4] Z. Bozkurt, İ. Gök, Y. Yayl, F.N. Ekmekçi, A New Approach for Magnetic Curves in Riemannian 3D-manifolds, J. Math. Phys., 55, (2014), 1-12. https://doi.org/10.1063/1.4870583

[5] C.L. Bejan, S.L. Druta-Romaniuc, Walker Manifolds and Killing Magnetic Curves, Differential Geometry and its Applications, 35, (2014), 106-116. https://doi.org/10.1016/j.difgeo.2014.03.001

[6] D. Das, N. Goswami, V.N. Mishra, Some results on the projective cone normed tensor product spaces over Banach algebras, Boletim da Sociedade Paranaense de MatemÁ̈itica, 38(1), (2020), 197-221. DOI: 10.5269/bspm.v38i1.36450. https://doi.org/10.5269/bspm.v38i1.36450

[7] S.L. Druta-Romaniuc, M.I. Munteanu, Killing Magnetic Curves in a Minkowski 3-Space, Nonlinear Anal-Real., 14, (2013), 383-396. https://doi.org/10.1016/j.nonrwa.2012.07.002

[8] S.L. Druta-Romaniuc, M.I. Munteanu, Magnetic Curves Corresponding to Killing Magnetic Fields in $E^{3}$, Journal of Mathematical Physics, 52, (2011), 113506. https://doi.org/10.1063/1.3659498

[9] P.P. Kruiver, M.J. Dekkers, D. Heslop, Quantification of Magnetic Coercivity Componets by the Analysis of Acquisition, Earth and Planetary Science Letters, 189(3-4), (2001), 269-276. https://doi.org/10.1016/s0012$821 x(01) 00367-3$

[10] M. Kulahci, F. Almaz, Some Characterizations of Osculating in the Lightlike Cone, Bol. Soc. Paran. Math., 35(2), (2017), 39-48. https://doi.org/10.5269/bspm.v35i2.26227

[11] D.N. Kulkarni, U. Pinkall, Conformal Geometry, Friedr. Viewey and Son, (1988).

[12] H. Liu, Curves in the Lightlike Cone, Contribbutions to Algebra and Geometry, 45(1), (2004), 291-303. 
[13] H. Liu, Q. Meng, Representation Formulas of Curves in a Two-and Three-Dimensional Lightlike Cone, Results Math., 59, (2011), 437-451. https://doi.org/10.1007/s00025-011-0108-y

[14] D. Lerner, Lie Derivatives, Isometries, and Killing Vectors, Department of Mathematics, University of Kansas, Lawrence, Kansas 66045-7594, (2010).

[15] L.N. Mishra, On existence and behavior of solutions to some nonlinear integral equations with Applications, Ph.D. Thesis (2017), National Institute of Technology, Silchar 788 010, Assam, India.

[16] V.N. Mishra, Some Problems on Approximations of Functions in Banach Spaces, Ph.D. Thesis (2007), Indian Institute of Technology, Roorkee 247 667, Uttarakhand, India.

[17] M.I. Munteanu, A.I Nistor, A Note Magnetic Curves on $S^{2 n+1}$, C. R. Acad. Sci. Paris, Ser. I, 352, (2014), 447-449. https://doi.org/10.1016/j.crma.2014.03.006

[18] L.I. Piscoran, V.N. Mishra, Projectively flatness of a new class of ( $\alpha, \beta)$-metrics, Georgian Math. Journal, (2017), DOI: $10.1515 / \mathrm{gmj}-0034$. https://doi.org/10.1515/gmj-2017-0034

[19] L.I. Piscoran, V.N. Mishra, S-curvature for a new class of ( $\alpha, \beta)$-metrics, Revista de la Real Academia

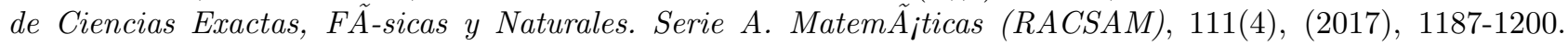
https://doi.org/10.1007/s13398-016-0358-3

[20] L.I. Piscoran, V.N. Mishra, The variational problem in Lagrange spaces endowed with a special type of $(\alpha, \beta)$ metrics, Filomat, 32(2), (2018), 643-652. https://doi.org/10.2298/fil1802643p

[21] T. Sunada, Magnetic Flows on a Riemannian Surface, in: Proceedings of KAÍIT Mathematics workshop, (1993), 93-108. 